\title{
Preliminary results from the UMBRELLA project: A volunteer-mentoring initiative for socially disadvantaged university students
}

\author{
Michalis Vitoulis $^{(1)}$ Vasilis Grammatikopoulos $^{(1)}$, Evridiki Zachopoulou ${ }^{(1)}$, Athanasios \\ Gregoriadis $^{(2)}$ and the Research academic team of the "Umbrella" project \\ (1) International Hellenic University, Greece \\ (2) Aristotle University of Thessaloniki, Greece \\ * The project is funded by the European Union under the Operational Programme 'Human Resources \\ Development, Education and Lifelong Learning'
}

\begin{abstract}
The Umbrella project is a volunteer-based initiative of the International Hellenic University funded by the European Union under the partnership agreement 2014-2020 (project code: 5048170). The main goal of the 4-year project is to offer support to disadvantage students at a socio-emotional, academic, and practical level for helping them complete their undergraduate studies. The current study presents some preliminary results from the impact the Umbrella project during a two-year period (12/2018 till 12/2020). A total of 89 students at the International Hellenic University who received support from the Umbrella project participated in the study. A 17 items questionnaire was administered for the evaluation of the impact and the quality of the provided services. Results revealed that socially disadvantaged students reported a positive impact from their participation in the Umbrella project, both on their attendance and their academic progress. Moreover, results highlight the value and importance of volunteer-mentoring initiatives, especially in times of social and economic crises.
\end{abstract}

Keywords: peer-mentoring, disadvantaged students, academic attrition, higher education 